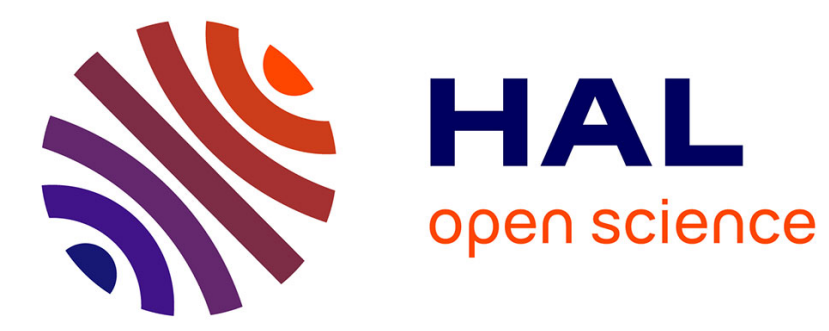

\title{
L'utilité et la rationalité dans les pratiques religieuses des femmes islamiques en Iran
}

Fariba Adelkhah

\section{To cite this version:}

Fariba Adelkhah. L'utilité et la rationalité dans les pratiques religieuses des femmes islamiques en Iran. L'Homme et la Société, 1991, 99 (99-100), pp.185-195. 10.3406/homso.1991.2548 . hal-01009639

\section{HAL Id: hal-01009639 \\ https://hal-sciencespo.archives-ouvertes.fr/hal-01009639}

Submitted on 18 Jun 2014

HAL is a multi-disciplinary open access archive for the deposit and dissemination of scientific research documents, whether they are published or not. The documents may come from teaching and research institutions in France or abroad, or from public or private research centers.
L'archive ouverte pluridisciplinaire HAL, est destinée au dépôt et à la diffusion de documents scientifiques de niveau recherche, publiés ou non, émanant des établissements d'enseignement et de recherche français ou étrangers, des laboratoires publics ou privés.

\section{(이)(\$)}

Distributed under a Creative Commons Attribution - NonCommercial - NoDerivatives| 4.0 


\section{L'utilité et la rationalité dans les pratiques religieuses des}

\section{femmes islamiques en Iran}

In: L Homme et la société, N. 99-100, 1991. Femmes et sociétés. pp. 185-195.

\section{Abstract}

Fariba Adelkhah, The Utility and Rationality of Religious Practices among Islamic Women in Iran

The study of the motivations which lie behind certain practices relative to women in post-revolutionary Iran indicate the existence of a space-time matrix within which conjunctival factors are determinant The examples of the gift and alms illustrate new interrogations about religious activities and the motivations associated with them. Such study is not intended to question the intensity or legitimacy of religious traditions, but rather to explore areas of liberty which exist within such traditions.

Citer ce document / Cite this document :

Adelkhah Fariba. L'utilité et la rationalité dans les pratiques religieuses des femmes islamiques en Iran. In: L Homme et la société, N. 99-100, 1991. Femmes et sociétés. pp. 185-195.

doi : $10.3406 /$ homso.1991.2548

http://www.persee.fr/web/revues/home/prescript/article/homso_0018-4306_1991_num_99_1_2548 


\section{L'utilité et la rationalité dans les pratiques religieuses des femmes islamiques en Iran}

Fariba ADEL KHAH

La Révolution iranienne de 1979 n'a pas simplement signifié une transformation radicale du pouvoir politique. Elle a cristallisé des contestations sociales et religieuses qui se sont poursuivies après la consolidation du nouveau régime. Les femmes islamiques ont constitué une dimension symbolique importante de cette période parce qu'elles ont été présentes dans les manifestations durant les moments les plus agités mais aussi parce qu'elles exprimaient une tentative originale d'articulation du religieux et du social dans l'espace féminin.

La nouveauté au moins apparente de ce phénomène ne doit pas faire oublier que la révolution n'a pas annulé en quelques mois certaines dynamiques sociales déjà à l'œuvre sous l'empire Pahlavi. Bien au contraire pourrions-nous dire. Pour mieux saisir l'originalité de ces femmes, il est intéressant d'analyser certaines pratiques religieuses non obligatoires comme les vœux et les aumónes et leurs éventuelles contestations : cela permet de mieux saisir le mode d'articulation des champs religieux et social des femmes islamistes et l'importance de certaines aspirations qui les structurent.

Sans qu'elles soient expressément réservées à l'un des deux sexes, ces pratiques semblent être un quasi-monopole féminin dans la mesure où elles suscitent, de façon régulière, des engagements et des mobilisations spécifiques chez les femmes islamiques. Les hommes, quant à eux, doivent assurer sans faille les frais que ces pratiques supposent en général. Ce soutien, sans jamais être mis en cause par les femmes, n'est point contesté par les hommes. Ainsi la réalisation de telles activités doit bénéficier d'un accord tacite ou préalable au sein du couple. Mais il s'agit moins d'une situation de dépendance par rapport aux hommes que d'initiative souvent individuelle des femmes. La transformation du rapport au religieux ne concerne pas le statut de ces pratiques, leur conception interne, mais le sens et la nature de l'engagement et sa visée générale.

Nous ne nous intéresserons pas à la genèse de ces activités, ni à leurs liens étroits et/ou distendus aux textes religieux. En prenant comme cadre d'analyse les discours tenus par les femmes islamistes sur celles-ci, nous allons concentrer notre étude sur les raisons qui fondent leur permanence 
et/ou leur contestation ainsi que sur le buts et finalités qu'elles doivent atteindre, les sociabilités et dynamiques nouvclles qu'elles autorisent.

Ce travail n'entend nullement juger l'intensité du sentiment religieux des femmes islamistes; il tente d'avancer des éléments d'analyse sur les formes de leur contestation religieuse en récusant d'une part l'explication culturaliste qui s'est constituée autour du shi'isme, d'autre part celle d'une manipulation utilitaire de la religion qui permettrait d'accéder à la modernité et/ou au pouvoir, mais qui ne serait réservée qu'aux plus démunis au sens propre et figuré...

Le vœu et l'aumône sont considérés, dans ce travail, comme étant des modes de communication des femmes avec le monde divin. Néanmoins ils se distinguent des devoirs religieux par leur caractère non obligatoire. Il s'agit donc d'initiatives volontaires, qui procèdent d'un engagement personnel. Si l'on veut parler d'obligation, il faut alors inverser la logique : c'est poussée par certaines exigence individuelles ou des nécessités propres (souvent sociales) que la croyante mobilise les signes religieux pour quémander auprès de Dieu quelque faveur, le "bien-être".

Cependant, réduire d'une façon trop rapide et directe ces activités religieuses à la simple réalisation de demandes ou de vœux individuels serait tout à fait erroné. Les femmes islamiques manifestent à l'égard de ces pratiques un certain activisme plutôt qu'un attentisme ou une passivité ; de plus, l'éthique traditionnelle iranienne s'oppose fermement à l'individualisme. Avec la Révolution, cette attitude s'est renforcée en se valorisant chez les femmes islamistes car elle traduit une volonté d'étendre les activités religieuses au-delà de la sphère individuelle et de leur reconnaître un caractère social qui, seul, semble être en mesure, dans le discours des interviewées, d'expliquer la soumission sans faille au divin. En toute première approximation - mais nous verrons que le sens en est bien plus profond - il faut relever le caractère de revanche d'un tel discours collectif et solidaire par rapport à la situation vécue dans l'Iran pré-révolutionnaire, sans oublier la volonté du gouvernement actuel d'institutionnaliser certaines pratiques religieuses à caractère social (comme la quête)...

Ainsi, il importe de noter, à partir de ce que disent ces femmes islamistes, combien leur comportement, leurs façons d'agir pour ce qui touche aux activités religieuses, sont déterminés non seulement par leur foi mais aussi par leur statut d'actrices sociales responsables ou encore, depuis la Révolution, par leur nouvelle conscience politico-nationale en devenir. Autrement dit, il faut souligner une nouvelle fois combien le cadre de ces activités dépasse largement la motivation individuelle (et individualiste), combien aussi elles doivent être interprêtées comme des dynamiques au-delà de tout utilitarisme primaire ; rien donc ne serait plus faux que d'en faire la $(n+1)$ ème expression de la pauvreté et de l'inertie. Affectées quelquefois profondément par les changements postrevolutionnaires, elles suscitent de nouveaux lieux d'interrogation et de reflexion sur le religieux, le social et même le politique... 
Dans les deux cas, ces activités sont semblables a des engagements contractuels entre la créature et le Créateur à partir d'un acte volontaire de celle-ci : elle s'engage, en échange de la réalisation de ses vœux, à approfondir sa foi ou à répondre à l'exigence divine en effectuant une action particulière. Il y a donc, dans le même temps, une espèce de pression sur Dieu qui nâ̂t de l'absence de négociation sur l'engagement de chacun.

L'offre divine est, certes, une faveur souhaitee, mais elle ressemble davantage à une sorte de revendication qui est, alors, affirmé à travers l'action ou le niyyat (l'intention) de celle-ci. Il ne faudrait pas, non plus, ici, qualifier cette revendication d'individualiste, car, comme nous allons le voir plus loin, selon les femmes islamistes, presque toutes les actions menées sur le chemin de la foi islamique sont à caractère social.

Comment définissent-elles ces activités? "Ce sont des pratiques qui permettent d'établir une relation avec Dieu pour nos besoins.", expliquent quelques-unes tandis que d'autres, en plus grand nombre, soulignent plutôt leur aspect social : "Elles sont des prétextes islamiques pour aller au secours des pauvres". Une troisième attitude établit une liaison entre les deux premières : "Tout ce que nous réalisons pour la société, c'est comme si nous le réalisions pour Dieu. Être au service de l'autre, c'est être au service de Dieu car l'autre n'est que sa créature."1

Avant d'expliciter davantage l'ouverture au social qu'exprimeraient ces activités, je voudrais revenir sur quelques-uns de leurs aspects fondateurs et sur les stratégies adoptées par les croyantes.

\section{DON ET PRIEREE : LES DEUX COMPOSANTES DES ACTIVTTES RELIGIEUSES}

Le don et la prière sont les deux éléments sur lesquels se fondent ces activités religieuse. Ils sont complémentaires, parce qu'il est quelquefois difficile de les distinguer. Toutefois pour le sadaqât (aumônes) - comme le nom le suggère - le don tient une place essentielle, la prière est alors tout à fait secondaire ou inexistante de façon formelle. Pour les vœux la situation peut s'inverser dans certains cas, notamment lorsque leur réalisation est indépendante des autres. Mais pour les Iraniennes le vœu reste souvent conçu comme un don ou plus exactement un contre-don.

Lorsque la prière devient le pivot du vœu, c'est que deux types de situations se présentent habituellement; soit les femmes n'ont pas de ressources propres - ainsi les étudiantes - soit il y a une stratification, sans doute peu consciente mais tout à fait réelle, des vœux. Ainsi quand la

1. Ce lien n'est certes pas une spécificité de l'islam. Marc Augé a souligné dans le rapport des hommes au vodu "l'imbrication du monde des dieux et du monde des hommes (...), de la terre et du ciel dont la calebasse fermée propose une image et foumit un modèle effectivement utilisé dans l'activité rituelle" (cf. Le Dieu objet, Paris, Flammarion, 1988, p. 24). 
croyante espère avoir un enfant (en l'occurrence un garçon) ou une maison, le contre-don devient important. La réussite des enfants aux examens se situe bien plus bas dans ce classement. À la base de cette hiérarchisation, latente et relative, il y a souvent la difficulté d'accéder à certains buts plus que la nature même du vœu. Par exemple l'absence prolongée d'un fils basiji (mobilisé sur le front) pousse la mère à s'engager de façon de plus en plus importante avec le temps qui passe : on passe des prières au mouton égorgé, puis à la nappe d'Abolfazl $^{2} \ldots$ Mais jamais on ne sacrifie le tapis sur lequel on s'asseoit et la maison dans laquelle on habite ! Il y a ainsi une limite à tout sacrifice en dehors même de son caractère sacré3.

Don et contre-don dans les familles aisées sont bien plus fréquents, et pour cause, que dans les familles modestes. Cela n'est pas dû seulement à leurs possibilités économiques plus grandes mais correspond aussi à un moyen de distinction et de prestige social.

Enfin, la prière peut aussi - cela se passe souvent ainsi accompagner un engagement fondé sur le don. Elle apparaît alors sous plusieurs formes; cette diversité existe aussi pour le don qui est accompagné par une prière, conçue comme base de l'engagement.

S'il y a une différence entre ces deux catégories de pratiques, vœux et aumônes, elle se situe dans la double stratégie adoptée par les croyantes dès qu'elles sont engagées dans celles-ci. On peut parler à mon sens de deux sortes d'engagements : l'un relatif au vœu est "conditionnel", l'autre relatif aux sadaqât,est "direct". Pour les vœux, en effet, qu'ils soient initialement fondés sur la prière ou le don, la réalisation de l'engagement religieux reste conditionnée par une réponse positive de Dieu à la demande exprimée. Bref, on retrouve cette nature contractuelle de l'échange qui justifie l'expression "contre-don". Au contraire les sadaqât, eux, semblent suivre une logique inverse. Les femmes islamistes les qualifient d" "actions préventives" (ehtiyâti) ou de pratiques pour "écarter le mal" (raf'-e balâ). L'engagement de la croyante est effectif dès le vœu exprimé. Tel est le cas, par exemple, lorsqu'on égorge un mouton à la naissance d'un enfant pour qu'il ait une bonne santé, ou lorsque quelqu'un a échappé à une grave maladie ou à un grand danger. Les femmes parlent à ce propos de l'assurance (bimeh) : "J'ai assuré mon bébé dès sa naissance auprès de Hazrat-é Mahdi (le douzième Iman)". Cela illustre aussi l'une des remarques de Mauss qui met en rapport l'abondance des cadeaux au(x) Dieu(x) avec celle, désirée, des richesses pour les hommes ${ }^{4}$. Ces gestes peuvent se répéter à l'achat d'une voiture

2. La nappe d'Abolfazl est une cérémonie religıeuse autour d'une nappe où sont disposés des mets fins, des gâteaux, des fruits... Abolfazl est le cousin de l'Emâm Hossein (troisième Emân shi'ite, fils d'Ali); c'est avec ce demier qu'il est mort en martyr à Karbalâ.

3. Celle-ci tient aux conditions que rappelle François-André Isambert, pour qui une véritable théorie du sacré doit être "champ sémantique, espace symbolique, et système opératoire" (cf. Le sens du sacré, Paris, Minuit, 1982, p. 73).

4. Marcel Mauss, Sociologie et anthropologie, Paris, PUF, 1950, p. 165. 
ou après un accident (car il y a toujours un accident plus grave auquel on a échappe !). D'autres pratiques préventives ou de remerciement sont moins onéreuses et plus régulières : donner de l'argent à un pauvre (ou dans les coffrets dits de sadaqât mis en place à cette fin depuis la Révolution) au début de chaque mois lunaire, ou le jeudi soir.

Ces activités religieuses sont donc toujours le lieu d'une communication, de façon directe ou par l'intermédiaire des saints, avec le divin, d'un échange avec celui-ci, voire d'une multiplication des dons qui frise le "quasi-chantage" (pression morale) sur le Créateur. Ce sont les moments privilégiés d'une revendication concernant le bien-être individuel, sans pour cela ignorer le social, le public...

\section{L'UTIIITE : UNE OBSESSION PERMANIENTE}

Qu'il s'agisse du don, de la prière dans le cadre d'un vœu ou de l'aumóne, la réflexion des femmes islamistes, constamment et inlassablement, revient sur la notion d'utilité de la réalisation de cette activité religieuse. Cette utilité conduit même, dans certains milieux, à l'abandon de certaines pratiques traditionnelles au profit d'autres plus modernes et donc plus utiles. Toutcfois, il ne faudrait pas voir là une attitude unanime ; des pratiques traditionnclles, qualifiées d" "inutiles" se poursuivent aujourd'hui comme hier.

Comment s'expliquent ces divergences ? La notion d'utilité est présente chez toutes les femmes. C'est par clle que les femmes islamistes cherchent à rationaliser leurs pratiques religicuses et à prouver le bienfondé de celles-ci; c'est clle qui, sculc, justifie et valorise leur soumission à la religion, aux lois divines. Les débats concernent plutôt le champ d'interprétation de cette utilité ct les ambiguïtés qui en résultent. L'inutilité d'une pratique ne relève pas de sa nature intrinsèque mais plutôt d'une appréciation sur son usage ct son contcnu courant, i.e. la fin qu'elle vise, l'objectif qu'elle attcint. Cela permet de comprendre pourquoi règne une telle continuité, dans une certaine mesure, entre les activités religieuses d'hier et celles d'aujourd'hui malgre les critiques acerbes adressées aux pratiques traditionnclles.

Ainsi il s'agit, d'une part, de déterminer le niveau du discours sur l'utilité, qui est variable selon les individus et, d'autre part, d'en déterminer la cause. En effet, ce discours, avant même de rendre compte du contenu réel des pratiques, est revendiqué comme une marque d'affirmation et de distinction basćcs sur la connaissance (shenakht).

La rationalité de l'engagement religicux, sa valeur, se définissent ainsi à partir de l'utilité de l'action. Mais ccla est loin de servir de définition concrète et précise. Une étudiante en biologie, désirant valoriser l'utilité d'une prière par rapport au don, argumente : "Je trouve inutile de donner de l'argent à un mendiant car cela ne lui servira que pour un moment et son besoin resurgira une fois celte somme dépensée. Ce n'est 
d'ailleurs pas mon rôle mais celui de l'État de pourvoir aux besoins des pauvres. Ainsi mon engagement religicux se fait à l'aide d'actions qui me font avancer d'un pas de plus sur la voie divine, i.e. mon auto-formation sur le plan intellectuel".

Une action rationnelle est une action qui permet de renforcer la foi, en rendant le croyant plus conscient des raisons de sa soumission aux lois sacrées. La reconstruction de sa foi peut donc devenir un gage, un enjeu, dans la communication des musulmans avec Dieu. On critique ainsi violemment les attitudes mécaniques du croyant (dit) traditionnel : "Dire cent fois, mille fois Allaho akbar (Dieu est grand) - l'une des pratiques les plus courantes à l'aide d'un chapelet qu'on égrène - ne sert a personne, mieux vaut comprendre pourquoi on le dit" explique une étudiante. La simple lecture du Qorân qui est menée jusqu'à aujourd'hui par la grande majorité des croyants ne trouve pas non plus grâce à lcurs yeux; c'est l'exégèse qui foumit une alternative valorisée. Il faut coupler - beaucoup des interviewées le font - la lecture du Qorân et l'exégèse ; l'utilité se trouve dans une meilleure compréhension, un approfondissement et une extension du savoir religieux.

Il est intéressant de s'arrêter sur cette notion d'utilité dans ce cadre des pratiques religieuses basées sur la prière. En effet toute pratique répétitive est, a priori, taxée d'inutile, d'autant plus que, traditionnellement, la signification donné à celle-ci par les croyants est assez éloignée du sens réel du texte prononcé (souvent ignoré). On constate là ce rapport extraverti au divin, i.e. la relation au divin ne peut se créer que par un savoir acquis et non inné, qui semble caractériser le discours des femmes islamistes dans leur quête de rationalité. Cette attitude fait consensus parmi mes interviewées bien que les pratiques semblent quelque peu diverger. Le discours valorisant l'utilité de la prière par rapport au don est toutefois minoritaire et même peu representatif car, en pratique, le don reste une activité individuelle largement répandue alors que la prière - comme pratique régulière non obligatoire - est, elle, plutót une activité collective, autant dans sa forme traditionnelle qu'actuelle.

\section{LE CHAMP DUTHTEE DU DON}

Le don ne se suffit pas à lui-même pour fonder le discours utilitariste et rationaliste des femmes islamistes sur leurs pratiques religieuses. Il est un moyen dont l'efficacité et l'utilité se mesurent à deux niveaux : ce qu'on donne, à qui on donne. Ainsi le propre du don n'est pas seulement dans le fait de donner, d'autant plus que beaucoup de choses peuvent servir de don. 
Qu'est-ce qu'un don rationnel, utile ? Le débat ne se situe pas dans sa nature, en général de l'argent et de la nourriture ${ }^{5}$ mais dans son usage. Un don ne doit pas être un "gaspillage" (esrâf) et ne doit pas évoquer la superstition (khorâfât). Pourtant la majorité des femmes islamistes bien que réservée sur certaines pratiques opte pour un discours moins radical qui met l'accent sur leur inutilité. Cette réserve peut cependant s'expliquer.

D'une part, ces pratiques sont, pour certaines, attachées à une région - ajil-e moshkel gosh ${ }^{6}$ est connu et largement pratiqué des gens de Khorâsân, ce qui n'est pas le cas pour les autres régions d'Iran-, et souvent même à un groupe social - les hâji sont les seuls désirés le jour de la fête de sacrifice pour tuer un mouton. D'autre part, une jeune musulmane coupée des réseaux traditionnels de socialisation religieuse peut tenir aisément un discours radical, mais il en va autrement d'une femme religieuse qui reste liée à ces réseaux et qui ne peut concevoir une rupture avec son propre passé sans renier ou mettre en cause, en même temps, sa foi de croyante d'hier et risquer alors l'isolement dans la communauté. Enfin, il y a un positivisme islamique qui reconnaît aussi une expérience individuelle aux dépens de condamnations trop radicales : "Les gens arrivent à établir une relation privilégiée avec le divin par des moyens très variés."

Ce discours a indiscutablement un aspect très tolérant mais celui-ci va de pair avec une hiérarchisation (pas toujours explicitée) dans la communauté des croyants : il y a celles/ceux qui ont su ou pu approfondir leur foi, la développer à l'aide de la connaissance et celles/ceux qui n'ont pu le faire. Ainsi l'une de mes enquêtées affirme : "Je ne peux pas dire que ces pratiques soient des superstitions. Moi-même, j'y participais avec ma connaissance d'alors car elles me paraissaient être les seuls moyens possibles d'établir une relation avec Dieu et d'exprimer ma foi et ma fidélité. Ainsi j'obtenais à travers elles ce que je cherchais. Aujourd'hui je sais que certaines d'entre elles n'ont aucune utilité, aussi je les ai laissées." Une autre femme islamiste, se reférant de façon assez libre à un verset coranique, dit que Dieu n'exige du croyant que ce dont il est capable (le savant et l'ignorant, le sage et le fou, l'instruit et l'illettré ne sont pas tenus d'exprimer leur foi de la même façon...).

Ce discours de distinction revendiqué autant par rapport à un passé personnel perçu comme pétri d'ignorance que par rapport aux autres croyantes restées dans un cadre contreversé ne peut pourtant avoir des effets radicaux, notamment, éliminer les pratiques "inutiles". En effet,

5. La nourriture se présente sous différentes formes : viande fraîche, mets sales, très souvent potages, pâtisseries comme le Halvâ à base de farine, d'huile et de sucre ou le Sholeh zard à base de riz, de sucre, d'amandes et de safran.

6. Cette pratique consiste à distribuer des friandises à la mosquée ou lors d'une réunion religieuse.

7. On désigne ainsi ceux qui ont effectué le pèlerinage à La Mecque. 
outre les motifs évoqués plus haut, il y a le contexte socio-politique de l'Iran où la sacralisation du pouvoir et la politisation de la religion ont suscité des réactions divergentes de soutien mais aussi de retenue. Autrement dit, la prise de pouvoir par le clergé ne diminue guère les divergences inhérentes à la gestion du pouvoir. Elle se traduit par des tensions qui apparaissent au cœur des dispositifs de transmission du sacré. L'unité de parole, attribut fondamental du sacré, cède la place à la diversité des possibles...

À qui doit profiter le don? Bonne action que celle de donner mais une dernière condition s'impose : il faut encore que ce soit à ceux qui en ont un réel besoin, les mostaz'afin (les déshérités), certes, mais pas les mendiants ! "Je ne donne rien à un homme qui tend la main et qui, ainsi, se déshonore simplement pour répondre à des besoins matériels." Ainsi, le besoin matériel qui est le fondement de l'utilité du don et légitime en quelque sorte les pratiques les plus courantes ne doit jamais être exprimé par les individus eux-mêmes mais doit être reconnu de façon permanente par le croyant lui-même, conscient qu'il est des problèmes de sa société. Le non-dit sur l'économique correspond plutôt à son omniprésence dans la conscience des croyants ; cependant, il faut souligner le poids de l'éthique qui pèse sur le statut de la pauvreté et qui pousse à la discrétion autant le pauvre que le donateur. Celui-ci intervient rarement en personne pour transmettre son don à un pauvre, encore moins si celui-ci réside dans son quartier car c'est le rôle des dignitaires religieux ou tout simplement des hommes de confiance du quartier (mo'tamed-e mahal). C'est ce qu'on appelle le don indirect voisinant avec le don direct ou, quelquefois, s'opposant à celui-ci.

À un niveau plus immédiat, à qui profite, et à qui doit aussi profiter, selon les voeux des femmes islamistes, le don du croyant ? Chaque famille a, un peu, ses pauvres de confiance, ses mostaz'afin, ses nombreux seyyed (descendants du Prophète) qui, s'ils sont dans le besoin, sont prioritaires. Il y a aussi le sud de Téhéran, lieu des va-nupieds ( $p a$ berhneha) connus de tous hier comme aujourd'hui dont le gouvernement en place se veut le représentant. Il ne faut pas non plus oublier les mosquées dont les responsables - souvent les délégués de grands dignitaires religieux - se chargent de la collecte de ces dons et de leur gestion à des fins bien sûr islamiques.

Enfin, depuis la Révolution, "les coffrets de secours de l'Imâm, (sandoqhaye emdad-e emâm) sont à la disposition, ó combien aisée, des donateurs ${ }^{8}$. Leur nouveauté réside dans le développement des dons indirects mais, aussi et surtout, des dons impersonnels. Il s'agit de dons tout a fait anonymes : le croyant se trouve seul face au coffret. Nous allons voir que c'est dans cet acte que réside la pureté et la plénitude de

8. Un spécimen de ce coffret en miniature est distribué aux familles grâce aux mosquées de quartier ou aux diverses fondations gouvemementales. 
sens du don auxquelles quelques-unes de mes interviewées sont fort sensibles.

S'il y a développement des dons indirects et anonymes, le don direct est loin de céder de la place sur le champ des pratiques religieuses et n'a rien perdu de son attrait. Des sommes considérables sont, aujourd'hui plus que jamais, dépensées dans ce cadre pour des personnes qui ne sont pas toujours démunies. Ainsi en est-il du mouton égorgé, très souvent partagé parmi les voisins, les amis, ou de la nappe d'Abolfazl, ou encore la distribution de nourriture toujours dans le quartier du donateur.

Ces pratiques sont souvent durement critiquées par certaines femmes islamistes pour qui elles ne sont que gaspillage inutile : "Aujourd'hui le mouton ne profite au mieux qu'aux voisins qui ne sont d'ailleurs pas dans le besoin, sinon ce sont les congélateurs, qui sont devenus les nouveaux mostaz'af'. La critique ne se limite pas au gaspillage mais questionne l'usage que les donateurs en font, le profit tout à fait personnel qu'ils s'efforcent d'en tirer. "Je prefere donner aux "coffrets de secours", cela me fait plus réfléchir sur la nature réelle de ma pratique ; bien loin de moi l'idée d'imiter celles qui en usent comme un moyen de distinction sociale ou de satisfaction personnelle."

Certaines ont un point de vue plus retenu. Bien qu'elles reconnaissent la validité des accusations, elles préfèrent donner la priorité au don, quelles que soient les intentions qui le guident. Seul compte le bénéfice que peut en recueillir le pauvre. "Que les riches donnent aux pauvres, c'est cela qui compte pour moi, le reste c'est à Dieu d'en juger". Certaines activités religieuses très contestées peuvent être réappropriées, comme, par exemple, la nappe de Abolfazl. Certaines femmes vont réduire les dépenses perçues comme inutiles, ou freiner le gaspillage afin de rationaliser cette cérémonie ; d'autres vont au contraire justifier la richesse des mets par le respect dû à Abolfazl.

Mais l'accent mis sur l'utilité de l'action n'exclut pas des pratiques dont la légitimité utilitaire est réduite mais qui sont très valorisées par le croyant. On parle alors non plus de l'action utile ('amal-e mofid) mais d'action pieuse ('amal-e kheir, 'amal-e savâb). C'est la bonne intention du donateur qui semble ainsi jugée. "Ma voisine s'engage dans le cadre de ses vœux à m'acheter une paire de chaussettes. Elle obtient toujours ce qu'elle désire" dit une mère de famille et elle ajoute : "Je suis seyyed" et comme toute action en faveur des seyyed est une bonne action, nous sommes doublement dans l'action pieuse par la bonne intention du donateur et le statut religieux de la personne aidée. "Je paie le taxi de mes invitées chaque jeudi soir lorsque je vais avec elles sur la tombe de l'Emâm Reza" dit une citoyenne de Mashad qui demande à Dieu que le bienfait de son action aille à l'âme de sa belle-mère qu'elle n'a jamais vue?.

9. Il s'agit ici d'un don un mort qui est parmi les plus communs en Iran (et chez mes interviewees). Le jeudi soir est, souvent, un moment reservé au souvenir des morts. 
La recherche d'une rationalité des pratiques et de activités religieuses ne dissout pas un lien plus mystique ou plus magique au divin. Un ciel ensoleille en plein hiver pour la prière collective du vendredi qui se passe à l'air libre, l'avancée sur le front des soldats iraniens malgré la mobilisation des Occidentaux derrière l'Irak, deviennent des signes de l'appui divin à la Révolution iranienne, explique une diplômée des ÉtatsUnis. La famille est bien sûr un lieu privilégié de la reproduction d'une telle expérience. "Pour le mariage de mon frère nous avons fait un estekhareh (pratique qui consiste à ouvrir le Qorân et interpréter à la lumière des versets sur lesquels le Qorân s'ouvre l'appreciation divine concernant l'action entreprise). C'était négatif. Nous avons eu beaucoup de peine, aussi nous avons recommencé une deuxième puis une troisième fois avec toujours le même résultat. Malgré l'affection que nous avions pour la fille, nous avons renoncé au mariage. Aujourd'hui nous savons pourquoi ! Dieu soit loué ! Cette fille était une Mojâhed ${ }^{10}$ et nous l'ignorions."

\section{CONCLUSION}

Au terme de cette courte analysc, deux remarques s'imposent. Tout d'abord, il convient de constater l'absence de déterminisme du code sacré. Autrement dit, on ne peut guère réduire les pratiques des femmes islamiques, notamment celles issues du champ de la religiosité, à une simple réalisation des devoirs envers le transcendant. Affirmer que les normes ou les idées ne renvoient pas dans le quotidien à des structures figées est certes un truisme. Mais cela doit nous permettre, tout en mettant l'ardent sur l'ambivalence des pratiques religieuses en terre d'islam, de nous orienter vers une anthropologie dynamiste des sociétés islamiques et de refuser l'approche culturaliste qui s'est constituée autour du shi'isme depuis plusieurs années.

En second lieu, en relation directe avec ce travail, il faudrait s'interroger, sur le dynamisme propre de certains traits structuraux des conceptions des femmes islamistes, pour en saisir l'aspect novateur mais aussi les limitations.

La rationalisation a ses limites qui marquent la force des croyances populaires sur l'interprétation utilitariste et positiviste. Penser que toute action de grâce envers la famille du Prophc̀te portera des fruits, qu'il suffit de la bonne intention (niyat-e kheir) pour que toute action religieuse soit récompensée, ce sont là des croyances indiscutées qui dépassent tout concept de rationalité, ou d'utilité ${ }^{\prime \prime}$.

10. Les Mojâhed sont les membres d'une organisation d'opposition au gouvernement et aux clercs.

11. François-André Isambert note à ce propos qu'il "il est remarquable que, dans une sociêté où la vérité scientifique est donnée comme prototype de toute vérité, se soit développée, passé l'âge de l'intolérance scientiste, une sone de secteur libre de la vérité, où il soit donné de 
L'utilité, mesure de la rationalité dans la foi des femmes islamistes, renvoie à une fonctionnalité de la pratique religieuse. En cela, elle souligne un aspect important qui est celui d'une société en transition où le changement est ressenti comme inévitable et irréversible. In faut d'abord noter le caractère paradoxal de cette insistance sur l'utilité. Les femmes islamistes pratiquent intensivement la dissimulation de la pauvreté et ne souscrivent guère à une analyse économique des causes de la Révolution. Comment expliquer ce paradoxe si ce n'est en interrogeant la conjoncture actuelle de la société iranienne?

Il y a, bien sâr, la crise économique qui se traduit par des queues de rationnement et l'existence d'un marché noir. Pour beaucoup de familles, il est aujourd'hui difficile d'assumer le coût des rassemblements religieux ou de fêtes familiales. Bien des vœux ordinaires sont devenus difficiles à honorer. De cette situation naît une double frustration. Il n'est plus possible de "dépenser sans compter", ce qui était un aspect essentiel de la fête. Il n'est plus possible non plus d'échapper à ce nouvel ordre du quotidien, cet "ordre réel" qui proscrit ou limite fortement les rencontres avec la famille élargie et donc interdit de faire reconnaître son statut social, son prestige et son honneur.

Il y a aussi la conscience de cette crise dans une société où la logique communautaire se défait et où "le souci de soi" mène les individus à réfléchir aux normes sociales et aux intérêts collectifs. Ceci n'a rien de contradictoire avec les normes islamiques d'autant plus que l'efficacité, l'utilité et l'intérêt sont perçus comme la mesure même de la vérité et de la justice.

L'accent mis sur l'utilité renvoie donc à cette situation réelle et à la conscience de cette réalité vécue. C'est notamment pour cela que ce discours religieux, fondé sur une rationalité utilitariste, trouve une légitimité certaine puisqu'il est l'une des manifestations de cette conjoncture sociale si spécifique. De là à en conclure que l'utilité est un langage operatoire, i.e. qu'elle conduit à l'abandon de certaines conduites religicuses, à la transformation radicale d'autres, il y a un pas que nous ne franchirons pas...

(*) J'utilise de façon indifférenciéc les qualificatifs "islamique" et "islamiste" dans ce texte. Cette confusion me paraît justifiée par les implications sociales de la revendication religieuse des femmes islamistes.

développer des systèmes de conviction organisant non seulement les jugements de valeur mais des jugements de réalité sur la nature des êtres et de lcur destinée" (cf. François-André Isambert, op. cit., p. 271). 